\section{P1-S2.61 HIGH HIV RATES AMONG MEN WHO HAVE SEX WITH MEN IN JAMAICA DESPITE INCREASED PREVENTION EFFORTS}

doi:10.1136/sextrans-2011-050108.118

1J P Figueroa, ${ }^{2} \mathrm{~S}$ Weir, ${ }^{3} \mathrm{C} J$ Cooper, ${ }^{4} \mathrm{~L}$ Byfield, ${ }^{4} \mathrm{~S}$ Eastman, ${ }^{2} \mathrm{M}$ Hobbs, ${ }^{4} \mathrm{~J}$ Duncan. ${ }^{1}$ University of the West Indies, Kingston, Jamaica; ${ }^{2}$ University of North Carolina, Chapel Hill, USA; ${ }^{3}$ Epidemiology and Research Training Unit, Kingston, Jamaica; ${ }^{4}$ Ministry of Health, Jamaica

Background Adult HIV prevalence in Jamaica is $1.7 \%$ while rates among men who have sex with men (MSM) were $32 \%$ in a 2007 survey. Following this survey prevention interventions among MSM were increased. The objective of this study was to estimate HIV rates among MSM in 2011 and compare findings with the 2007 survey.

Methods An experienced research nurse attended prevention workshops for MSM as well as their social activities and invited MSM to participate in a cross sectional survey. MSM were interviewed and tested for HIV and other STIs. Sensitive behavioural data was interviewer administered and self-completed. Confidential results were given to participants with treatment as indicated. Ethical approval and informed consent was obtained. The 2011 survey is ongoing so results are preliminary.

Results 33\% of 157 MSM were HIV positive in 2011 compared with $32 \%$ of $201 \mathrm{MSM}$ in 2007. In 2011, 8 of 157 (5.1\%) MSM were positive for syphilis and $3.8 \%$ were indeterminate. 12 of 139 MSM $(8.9 \%)$ were positive for Chlamydia and $2(1.4 \%)$ had gonorrhoea. In 2007 HIV positive MSM were more likely to ever have a STI $(37.5 \%$ vs $19.0 \%, p=0.004)$ and to be receptive $(73.4 \%$ vs $59.1 \%, p=0.005)$. $33 \%$ of MSM reported sex with a woman in the past 4 weeks and $65 \%$ reported ever having sex with a woman. Nearly $60 \%$ of HIV positive MSM had not disclosed their status to their partner. MSM who were of low socio-economic status, ever homeless and victims of physical violence were significantly more likely to be HIV positive.

Conclusions HIV prevalence among MSM remains unacceptably high. MSM are more socially vulnerable than the general population. Many MSM reported having sex with a woman and not disclosing their HIV status to their partners. MSM are likely to act as a bridge for HIV into the general population. Prevention efforts among MSM need to be scaled up urgently and measures taken to reduce their social vulnerability including stigma and discrimination.

\section{Abstract P1-S2.61 Figure 1}

\section{P1-S2.62 FACTORS ASSOCIATED WITH HIGH-RISK PENETRATIVE SEX IN A COHORT OF TREATMENT EXPERIENCED HIV. POSITIVE MEN WHO HAVE SEX WITH MEN (MSM) IN BRITISH COLUMBIA}

doi:10.1136/sextrans-2011-050108.119

${ }^{1} \mathrm{~W}$ Michelow, ${ }^{2} \mathrm{G}$ Talany, ${ }^{1} \mathrm{~N}$ O'Brien, ${ }^{1} \mathrm{H}$ Wang, ${ }^{1} \mathrm{~J}$ Forrest, ${ }^{1} \mathrm{~A}$ Palmer, ${ }^{1} \mathrm{~J} S \mathrm{G}$ Montaner, ${ }^{2} \mathrm{R}$ Hogg. ${ }^{1} B C$ Centre for Excellence in HIVIAIDS, Canada; ${ }^{2}$ Simon Fraser University, Vancouver, Canada

Background In Canada, men who have sex with men (MSM) are disproportionately infected with HIV. Despite much evidence demonstrating the secondary preventive value of HAART, we have not fully capitalised on potential synergies between treatment and prevention. This study investigates high-risk sexual behaviour among treatment-experienced MSM in British Columbia, Canada. Methods We analysed data from the Longitudinal Investigations into Supportive and Ancillary Health Services (LISA) project on MSM (gay, bisexual, or reported sex with men in the 6 months prior to interview). High-risk sexual behaviour was penetrative anal or vaginal sex in the 6 months prior to interview with less than $100 \%$ condom use. Multivariate logistic regression was used to identify factors associated with high-risk penetrative sex.

Results Of 346 HIV-positive MSM, median age was 46 years, 268 (78\%) were currently on HAART, with 185 (54\%) having $\geq 350$ CD4 cells/ $\mu$ l. Ninety-eight (28\%) participants endorsed seeking out HIV-positive partners for sex, and 147 (43\%) reported being less likely to use a condom with an HIV-positive partner. In the regression model, HIV-positive MSM with a CD4 count $\geq 350$ cells $/ \mu 1$ were more likely to report unprotected penetrative sex (Adjusted OR [AOR] $=2.7 ; 95 \%$ CI 1.5 to 5.2), more likely to not use condoms with their HIV-positive partners ( $A O R=12.0$; 95\% CI:6.4 to 22.3), and less likely to report sex with anonymous partners in the past 6 months ( $\mathrm{AOR}=0.28 ; 95 \% \mathrm{CI} 0.1$ to 0.8 ). No significant associations were found between high-risk penetrative sex and current HAART use, viral suppression, or treatment adherence.

Conclusion HIV-positive MSM who report engaging in high-risk penetrative sex are more likely to be healthier (higher CD4 counts), to have unprotected sex with other HIV-positive partners (serosorting) and with known rather than anonymous sex partners. Despite healthier MSM reporting more high-risk sex, results suggest this risk-taking is discriminate and would not necessarily lead to more onward HIV transmission.

\section{P1-S2.63 ASSOCIATION BETWEEN AGE AND STI AMONG MEN WHO HAVE SEX WITH MEN (MSM) IN JAMAICA}

doi:10.1136/sextrans-2011-050108.120

${ }^{1} \mathrm{~S}$ Weir, ${ }^{2} \mathrm{P}$ Figueroa, ${ }^{3} \mathrm{C}$ Jones-Cooper, ${ }^{4} \mathrm{~T}$ Hylton-Kong, ${ }^{1} \mathrm{M}$ Hobbs, ${ }^{1} \mathrm{~K}$ Rich, ${ }^{3} \mathrm{~L}$ Byfield. ${ }^{1}$ University of North Carolina, Chapel Hill, USA; ${ }^{2}$ University of the West Indies, Kingston, Jamaica; ${ }^{3}$ Ministry of Health, Kingston, Jamaica; ${ }^{4}$ Epidemiology \& Research Training Unit, Kingston, Jamaica

Background In 2007, 201 men who have sex with men were recruited at social venues and through peer referral in five urban areas of Jamaica for an exploratory study of the prevalence of sexually transmitted infection in the population and identification of risk factors.

Objective To describe the association between age and current infection with HIV, syphilis, and chlamydia and explore reasons for the differences.

Methods MSM were invited to participate in a survey and testing. Urine and blood samples were collected at the time of the interview and later tested for evidence of HIV, syphilis, gonorrhoea and chlamydia. Men were given options about where to obtain results.

Results Of the three infections, HIV had the strongest positive association with age; syphilis a weaker positive association; and chlamydia a weak negative association. Risk factors varied somewhat by age and infection see Abstract P1-S2.63 Figure 1. For example, not using a condom in the past 4 weeks was associated with chlamydia infection among MSM age 15-19 but not for older MSM. Having a main sexual partner was associated with HIV infection for MSM age 15-19 but not for older MSM.

Conclusion Strategies for prevention of transmission need to be aware of differences in risk by age and by infection. 


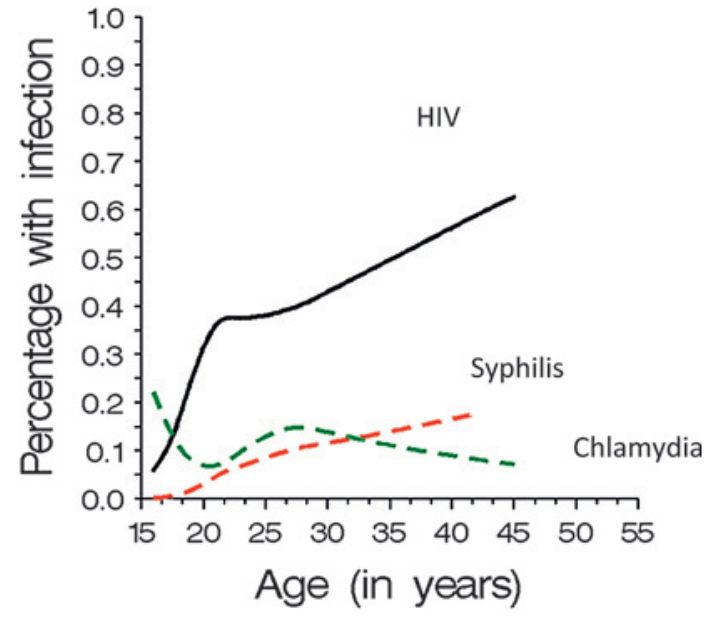

Abstract P1-S2.63 Figure 1

\section{P1-S2.64 DEMOGRAPHIC CHARACTERISTICS AND HIV RISK BEHAVIOURS AMONG MEN WHO HAVE SEX WITH MEN IN GUATEMALA CITY, GUATEMALA, 2010}

doi:10.1136/sextrans-2011-050108.121

${ }^{1} \mathrm{~B}$ Alvarez, ${ }^{1} \mathrm{~W}$ Miller, ${ }^{1} \mathrm{~F} \mathrm{M}$ Hernandez, ${ }^{2} \mathrm{~A}$ Alvarado, ${ }^{1} \mathrm{~S}$ Morales, ${ }^{2} \mathrm{G}$ Paz-Bailey. ${ }^{1}$ Centro de Estudios en Salud, Universidad del Valle de Guatemala, Guatemala; ${ }^{2}$ TEPHINET, Guatemala

Background In Central America, surveillance of HIV has been strengthened by regular and systematic collection of HIV, sexually transmitted infection, and behavioural data among most at risk populations. In Guatemala, men who have sex with men (MSM) are a highly vulnerable to HIV infection. Behavioural data are needed to help plan for prevention interventions in this group.

Methods Male residents of Guatemala City at least 18 years of age who self-reported having had anal sex with another man in the past 12 months were invited to participate in a face-to-face structured behavioural survey using respondent-driven-sampling (RDS) from September to December 2010. Participants who provided informed consent were administered a questionnaire that collected information on socio-demographics, risk behaviour, knowledge and attitudes, access to services, and stigma and discrimination, using personal digital assistants (PDA). Medians and IORs were calculated for continuous variables. Population proportions were calculated for categorical variables and adjusted for RDS-sampling weights using Respondent Driven Sampling Analysis Tool version 6.0 .

Results A total of 500 MSM enrolled in the study. Respondents self-identified as gay or homosexual (39.5\%), bisexual $(39.5 \%)$, heterosexual (11.5\%), and transvestite or transgender $(9.5 \%)$. Median age was 26 years (IOR 22-34 years). The majority of respondents $(79.5 \%)$ reported to have access to free condoms in the past 12 months. Consistent condom use with all male partners in last 12 months and during last sex was reported by $62.2 \%$ and $75.4 \%$, respectively. Overall, $44.3 \%$ reported having sex with a woman in the past 12 months. Consistent condom use with female sexual partners in the last 12 months and during last sex was reported by $46.1 \%$ and $66.0 \%$, respectively. The median number of female sexual partners reported in the last 12 months was 3 (IOR: 2-5). Ever paying for sex and paying for sex in the last 12 months was reported by $16.0 \%$ and $8.3 \%$, respectively; while $54.0 \%$ and $44.7 \%$ reported ever selling sex and in the last 12 months, respectively. Injection drug use in the last 12 months was reported by $0.8 \%$. Overall, $73.3 \%$ of respondents reported ever having an HIV test and $43.1 \%$ had been tested in the past 12 months.

Conclusions MSM in Guatemala engage in high risk behaviours that place them and their male and female partners at high risk for HIV infection. Innovative strategies are needed to promote condom use and access to HIV testing and counselling.

\section{P1-S2.65 CRACK/COCAINE USE AMONG MSM IN LATIN AMERICA: A MULTILEVEL ANALYSIS OF RDS STUDIES TO IDENTIFY DRUG USE PATTERNS AND ASSOCIATED FACTORS ACROSS CITIES}

doi:10.1136/sextrans-2011-050108.122

${ }^{1} \mathrm{~J} O$ Jacobson, ${ }^{1} \mathrm{M}$ Alonso-Gonzalez, ${ }^{2} \mathrm{D}$ Ramachandran, ${ }^{3} \mathrm{~S}$ Morales-Miranda, ${ }^{4}$ A Carballo-Dieguez, ${ }^{5} \mathrm{~J}$ Medrano, ${ }^{6} \mathrm{~T}$ Solano, ${ }^{1} \mathrm{M} \mathrm{D}$ Rosales-Perez. ${ }^{1}$ Pan American Health Organization, Bogota, Colombia; ${ }^{2} J o h n s$ Hopkins Bloomberg School of Public Health, Baltimore, USA; ${ }^{3}$ Del Valle University of Guatemala, Guatemala City, Guatemala; ${ }^{4}$ Columbia University, New York City, USA; ${ }^{5}$ Ministerio de Salud y Deportes, La Paz, Bolivia; ${ }^{6}$ Ministerio de Salud de Costa Rica, San Jose, Costa Rica

Background In Latin America (LA), men who have sex with men (MSM) are the population most affected by HIV, crack/cocaine production and trafficking routes are well-established, and local consumption has risen sharply in recent years. Yet, drug use and associations with sexual risk behaviours among MSM remain largely unexamined LA despite being recognised as important drivers of HIV transmission in the USA. Variation in crack/cocaine consumption within LA is also unknown.

Methods Data from participants self-identifying as MSM and aged 18 years and older in recent (2006-2009) respondent-driven sampling (RDS) studies using similar survey methodologies in nine high-population cities in Argentina, Bolivia, Costa Rica, El Salvador and Honduras $(\mathrm{N}=2081)$ were pooled and analysed in a multilevel statistical framework to increase statistical power to evaluate past 12-month crack/cocaine consumption (CC). CC prevalence was estimated by study site, adjusting for sampling weights incorporating personal network and population sizes. Logistic, multilevel models identified correlates of CC, adjusted for intraclass correlation within levels defined by study site and recruitment chain, and partitioned variance in CC among levels.

Results CC prevalence was $21.7 \%$ (95\% CI 17.4 to 26.7 ) in the pooled sample and varied significantly across cities from $0.1 \%$ in Cochabamba, Bolivia to $44.5 \%$ in Ceiba, Honduras. In multivariate analysis, daily alcohol consumption (adjusted OR $[\mathrm{AOR}]=4.0$ ), sexual debut of $\leq 12(\mathrm{AOR}=3.1)$ and $13-17(\mathrm{AOR}=1.9)$ years old, self-identifying as transexual/transgender/transvestite (TTT) $(\mathrm{AOR}=2.2)$, age $18-22$ ( $\mathrm{vs}>40$ ) years $(\mathrm{AOR}=2.0)$, having a stable female partner $(A O R=1.8)$ and ever purchasing sex $(A O R=1.6)$ were associated with CC $(p<0.05)$. Significant city-level random effects expressed as $\mathrm{AOR}$ were $5.4(\mathrm{SE}=1.3)$ and $2.7(\mathrm{SE}=1.3)$ in Ceiba and San Pedro Sula, Honduras, respectively and $3.4(\mathrm{SE}=1.2)$ in Buenos Aires, Argentina. Of the total variance in CC, $29.3 \%$ was associated with unobserved differences among cities. Associations between CC and condom use at last sex, and recruitment chain, were nonsignificant $(\mathrm{p}>0.1)$.

Conclusions CC prevalence among MSM, and variation among cities in LA, are considerable. Associations with frequent drinking and sexual risk Behaviours likely compound HIV transmission risk among MSM and to female partners. Substance abuse prevention and treatment for MSM are needed, particularly among TTT, and in Honduras and Argentina. 